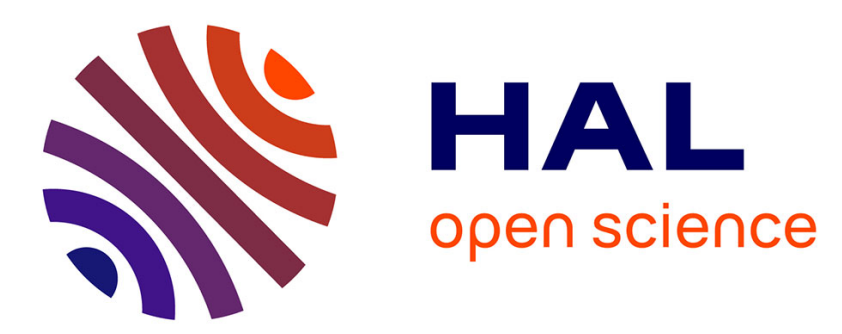

\title{
Theory and observation of electromagnetic ion cyclotron triggered emissions in the magnetosphere
}

Yoshiharu Omura, Jolene Pickett, Benjamin Grison, Ondřej Santolík, Iannis Dandouras, Mark Engebretson, Pierrette Décréau, Arnaud Masson

\section{- To cite this version:}

Yoshiharu Omura, Jolene Pickett, Benjamin Grison, Ondřej Santolík, Iannis Dandouras, et al.. Theory and observation of electromagnetic ion cyclotron triggered emissions in the magnetosphere. Journal of Geophysical Research Space Physics, 2010, 115, A07234 (13 p.). 10.1029/2010JA015300 . insu01180793

\section{HAL Id: insu-01180793 \\ https://hal-insu.archives-ouvertes.fr/insu-01180793}

Submitted on 28 Jul 2015

HAL is a multi-disciplinary open access archive for the deposit and dissemination of scientific research documents, whether they are published or not. The documents may come from teaching and research institutions in France or abroad, or from public or private research centers.
L'archive ouverte pluridisciplinaire HAL, est destinée au dépôt et à la diffusion de documents scientifiques de niveau recherche, publiés ou non, émanant des établissements d'enseignement et de recherche français ou étrangers, des laboratoires publics ou privés. 


\title{
Theory and observation of electromagnetic ion cyclotron triggered emissions in the magnetosphere
}

\author{
Yoshiharu Omura, ${ }^{1}$ Jolene Pickett, ${ }^{2}$ Benjamin Grison, ${ }^{3}$ Ondrej Santolik, ${ }^{3,4}$ \\ Iannis Dandouras, ${ }^{5,6}$ Mark Engebretson, ${ }^{7}$ Pierrette M. E. Décréau, ${ }^{8}$ and Arnaud Masson ${ }^{9}$ \\ Received 23 January 2010; revised 29 March 2010; accepted 1 April 2010; published 31 July 2010.
}

[1] We develop a nonlinear wave growth theory of electromagnetic ion cyclotron (EMIC) triggered emissions observed in the inner magnetosphere. We first derive the basic wave equations from Maxwell's equations and the momentum equations for the electrons and ions. We then obtain equations that describe the nonlinear dynamics of resonant protons interacting with an EMIC wave. The frequency sweep rate of the wave plays an important role in forming the resonant current that controls the wave growth. Assuming an optimum condition for the maximum growth rate as an absolute instability at the magnetic equator and a self-sustaining growth condition for the wave propagating from the magnetic equator, we obtain a set of ordinary differential equations that describe the nonlinear evolution of a rising tone emission generated at the magnetic equator. Using the physical parameters inferred from the wave, particle, and magnetic field data measured by the Cluster spacecraft, we determine the dispersion relation for the EMIC waves. Integrating the differential equations numerically, we obtain a solution for the time variation of the amplitude and frequency of a rising tone emission at the equator. Assuming saturation of the wave amplitude, as is found in the observations, we find good agreement between the numerical solutions and the wave spectrum of the EMIC triggered emissions.

Citation: Omura, Y., J. Pickett, B. Grison, O. Santolik, I. Dandouras, M. Engebretson, P. M. E. Décréau, and A. Masson (2010), Theory and observation of electromagnetic ion cyclotron triggered emissions in the magnetosphere, J. Geophys. Res., 115, A07234, doi:10.1029/2010JA015300.

\section{Introduction}

[2] Waves in Earth's space environment known as Pc1-2 pulsations (with frequencies from 0.1 to $5 \mathrm{~Hz}$ ) have long been observed both on the ground [Troitskaya, 1961; Tepley, 1961] and in space (e.g., by satellites such as GEOS-2 [Roux et al., 1982], AMPTE CCE [Anderson et al., 1992a, 1992b], Viking [Erlandson et al., 1990, 1996; Mursula et al., 1997], Freja [Mursula et al., 1994], Polar

\footnotetext{
${ }^{1}$ Research Institute for Sustainable Humanosphere, Kyoto University, Kyoto, Japan.

${ }^{2}$ Department of Physics and Astronomy, University of Iowa, Iowa City, Iowa, USA.

${ }^{3}$ Institute of Atmospheric Physics, Academy of Sciences of the Czech Republic, Prague, Czech Republic.

${ }^{4}$ Faculty of Mathematics and Physics, Charles University in Prague, Prague, Czech Republic.

${ }^{5}$ Centre d'Etude Spatiale des Rayonnements, Université de Toulouse, Toulouse, France.

${ }^{6}$ UMR 5187, CNRS, Toulouse, France.

${ }^{7}$ Department of Physics, Augsburg College, Minneapolis, Minnesota, USA.

${ }^{8}$ Laboratoire de Physique et Chimie de l'Environnement et de l'Espace, UMR 6115, Université d'Orléans, CNRS, Orleans, France.

${ }^{9}$ Science Operations Department, European Space Agency, Noordwijk, Netherlands.

Copyright 2010 by the American Geophysical Union. 0148-0227/10/2010JA015300
}

[Mursula et al., 2001; Arnoldy et al., 2005], and Cluster [Engebretson et al., 2007]). These waves are now known as electromagnetic ion cyclotron (EMIC) waves, and are understood to originate as a result of instabilities in the ion distributions that populate Earth's magnetosphere. Pitchangle scattering by EMIC waves in the outer radiation belt can induce significant loss of relativistic electrons under certain conditions [e.g., Lorentzen et al., 2000; Summers and Thorne, 2003; Summers et al., 2007].

[3] The most common class of Pc1 waves observed at mid- and low latitudes on the ground, so-called "pearl pulsations," is characterized by a sequence of highly structured wave packets with a repetition period on the order of minutes. Many early ground-based studies of Pc1-2 waves noted their highly structured, dispersive appearance in Fourier spectrograms, and attributed the observed temporal repetition to the existence of bouncing wave packets that, although generated near the magnetic equator and guided along the magnetic field, would reflect from the ionosphere and return equatorward to be re-amplified (e.g., as summarized by Kangas et al. [1998]).

[4] However, several satellite studies have called that picture into question (e.g., as reviewed by Fraser et al. [2006] and Mursula [2007]). The repetitive nature of the waves observed in space can in many cases be attributed to modulation by simultaneously observed longer period 
waves, and observations of the dispersive structure in space are quite rare. In addition, all observational studies of the propagation of these waves in space have shown unidirectional propagation of wave energy toward the ionosphere, and never away from it. Loto' aniu et al. [2005], who provided the most comprehensive recent observational study of this issue, used both magnetic and electric field data from the CRRES satellite in the $L$ range from 3.5 to 8.0 to determine Poynting vector directions for all Pc1-2 wave events observed during that spacecraft's $\sim 15$-month lifetime. They found unidirectional energy propagation away from the equator for all events located above $11^{\circ}$ MLAT, but bidirectional propagation, both away and toward the equator, for events observed below $11^{\circ}$ MLAT. Observations of propagation by Erlandson et al. [1990, 1996] using Viking satellite data at $\sim 3 R_{E}$ altitude far from the magnetic equator also showed purely downward propagation (toward the ionosphere), as did those by Mursula et al. [2001] and Arnoldy et al. [2005] using Polar satellite data near $25^{\circ}$ and $-22^{\circ} \mathrm{MLAT}$, respectively, and by Engebretson et al. [2007] using data from Cluster above $12^{\circ}$ MLAT.

[5] These studies suggest that the few instances of dispersive, intermittent Pcl waves observed in space cannot be attributed to the same mechanism that generates the dispersive, repetitive pearl structures observed so commonly on the ground. A recent study of Cluster data by Pickett et al. [2010] reports the observation of coherent rising tone emissions that emerge from nearly constant frequency EMIC waves. We believe the rising tones shown in Figure 8 of Mursula [2007] may be examples of this same phenomenon, which can be denoted as EMIC triggered emission, and for which we provide a quantitative physical model.

[6] Figure 1 displays about $1 \mathrm{~min} 20 \mathrm{~s}$ of Cluster 4 data on the 30th of March 2002. Figures 1a and $1 \mathrm{~b}$ display the magnetic and electric field fluctuation spectrograms measured respectively by the STAFF-SC [Cornilleau-Wehrlin et al., 2003] and the EFW [Gustafsson et al., 2001] instruments. Figures 1c and 1d show wave parameters estimated for the most energetic part of the magnetic fluctuation spectrogram with the Prassadco software [Santolik et al., 2003a]. From Figures $1 \mathrm{a}$ and $1 \mathrm{~b}$ one can notice an almost constant electromagnetic activity at about $1.5 \mathrm{~Hz}$, the Pc1 waves, and an electromagnetic rising tone emission starting from the Pc1 waves up to $3 \mathrm{~Hz}$. In Figures $1 \mathrm{c}$ and $1 \mathrm{~d}$, the magnetic fluctuations are analyzed in the polarization plane given by the singular value decomposition (SVD) method. Figure 1c shows that the sense of polarization (defined in equation (4) of Santolik et al. [2001]) is clearly negative for the rising tone, indicating the left-handed polarization. The sense of polarization of the Pcl waves is not well defined (both positive and negative values). It can be either indicative of a linear polarization or of no polarization. A low coherency value [Santolik and Gurnett, 2002; Santolik et al., 2002] in Figure 1d indicates that there are random phase shifts between the two components in the polarization plane. The high coherency level of the rising tone is a confirmation of its high degree of polarization. The left handed electromagnetic rising tone emission has a different origin. Pickett et al. [2010] demonstrate that the rising tone emission arrives from the magnetic equator.

[7] The spectra show the bursty nature of the emissions rising to higher frequencies. This bursty nature cannot be explained by the linear theory. The linear growth rate due to the temperature anisotropy of energetic protons maximizes near the helium cyclotron frequency of the lower helium band and the cut-off frequency of the upper proton band. Further, the linear growth rate decreases as the frequency approaches the proton gyrofrequency [Gendrin et al., 1984]. The bursty nature of the EMIC emissions requires much higher growth rates than the linear growth rates in the higher frequency range. A similar bursty wave growth is often observed in the inner magnetosphere as whistler-mode chorus emissions in a much higher frequency range [Santolik et al., 2003b]. Trakhtengerts and Demekhov [2007] applied the backward wave oscillator (BWO) model to Pc1 pulsations. The BWO model has also been applied to whistler-mode chorus emissions. In this model, a step-like velocity distribution is assumed, which causes an absolute cyclotron instability around the magnetic equator.

[8] As the generation mechanism of whistler-mode chorus emissions, Omura et al. [2008, 2009] have shown that there occurs a nonlinear absolute instability of coherent whistlermode waves due to the formation of an electromagnetic electron hole [Omura and Summers, 2006] in the velocity phase space. Since L-mode EMIC triggered emissions have certain characteristics quite similar to whistler-mode chorus emissions, we study the generation mechanism of them based on the nonlinear wave growth theory that assumes the formation of an electromagnetic proton hole.

[9] We first derive a set of wave equations for EMIC waves in section 2 . We then study the nonlinear dynamics of protons interacting with an EMIC wave in section 3 . We obtain the nonlinear growth rate of EMIC waves in section 4. Taking into account the inhomogeneous magnetic field around the equator, we determine a threshold for self-sustaining emissions of EMIC waves and obtain a set of nonlinear equations that describe an EMIC rising tone emission in section 5. In section 6 we compare the numerical solutions of the nonlinear equations with the observations by the Cluster spacecraft. In section 7 we present the summary and discussion.

\section{EMIC Wave Equations}

[10] We assume a coherent EMIC wave propagating parallel to a dipole magnetic field $\mathbf{B}_{0}$ of the inner magnetosphere. We measure a distance $h$ along the magnetic field line from the magnetic equator, and denote it as the $h$-axis with the direction of the static magnetic field. The wave fields are in the transverse plane containing the $x$ - and $y$-axes, where the coordinates $(x, y, h)$ form a right-handed system. We express the electric and magnetic field vectors of the wave with amplitudes $E_{w}$ and $B_{w}$ and phases $\psi_{E}$ and $\psi_{B}$ in the transverse plane by the complex numbers $\vec{E}_{w}=E_{w} \exp \left(i \psi_{E}\right)$ and $\vec{B}_{w}=B_{w} \exp \left(i \psi_{B}\right)$ in the complex plane, respectively. For an L-mode wave propagating in the positive $h$ direction, the frequency $\omega$ and wavenumber $k$ are defined by the following equations:

$$
\begin{gathered}
\omega=-\frac{\partial \Psi_{B}}{\partial t}, \\
k=\frac{\partial \Psi_{B}}{\partial h} .
\end{gathered}
$$


(a)

(b)
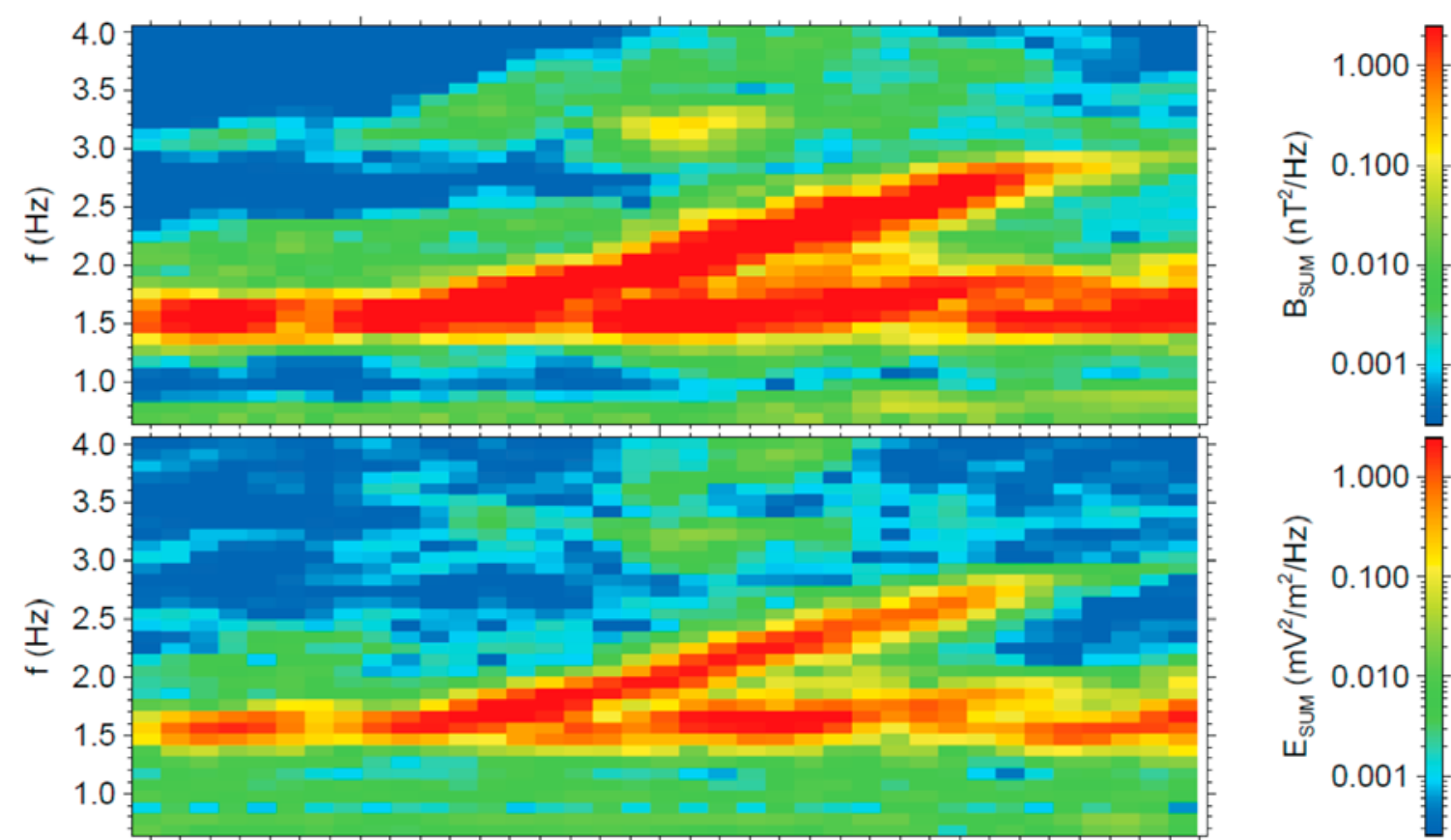

(c)

(d)
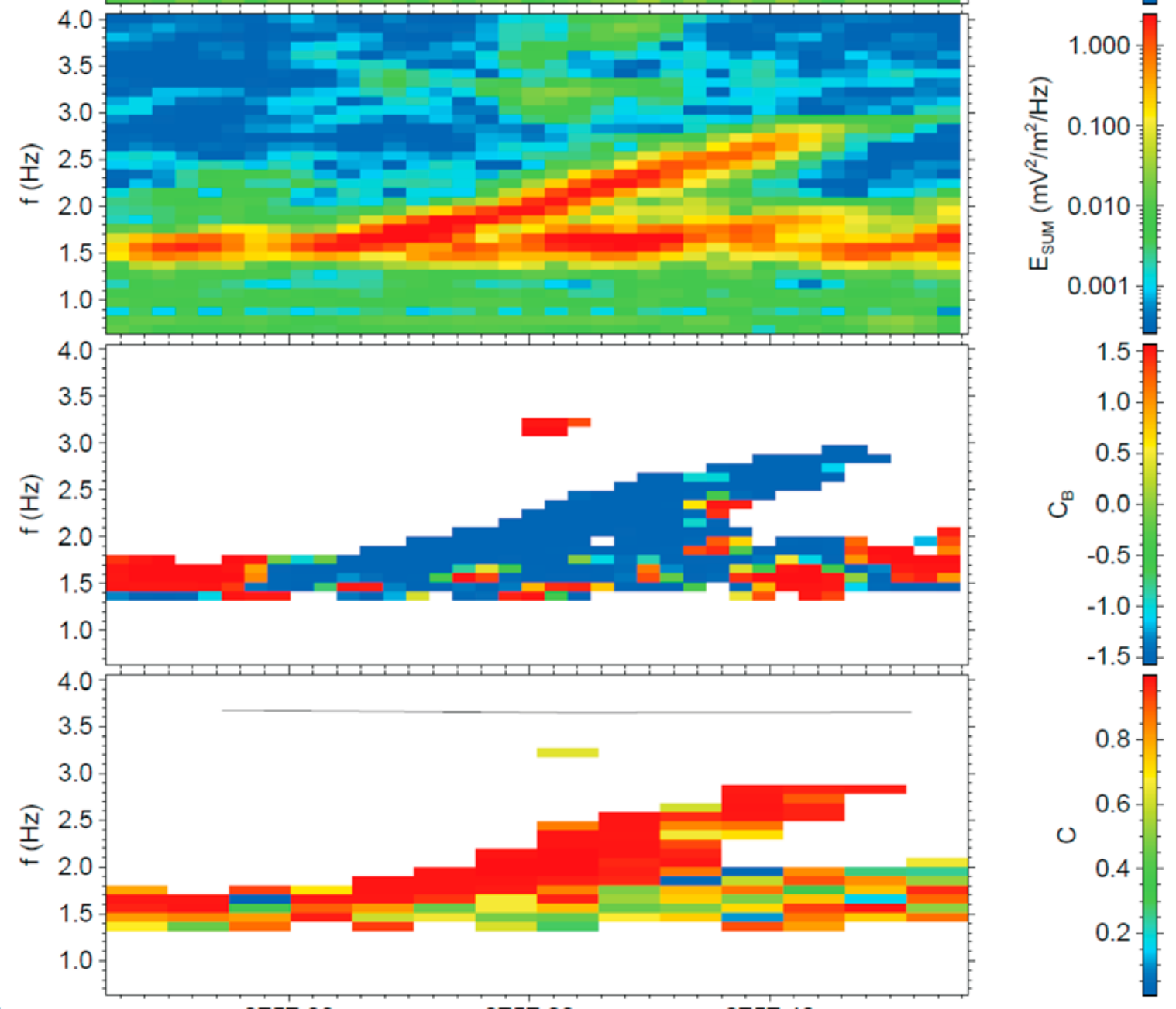

UT:
$X_{\text {GSE }}\left(R_{E}\right):$
$Y_{\text {GSE }}\left(R_{E}\right):$
$Z_{G S E}\left(R_{E}\right):$
MLT $(h):$

MLat (deg):

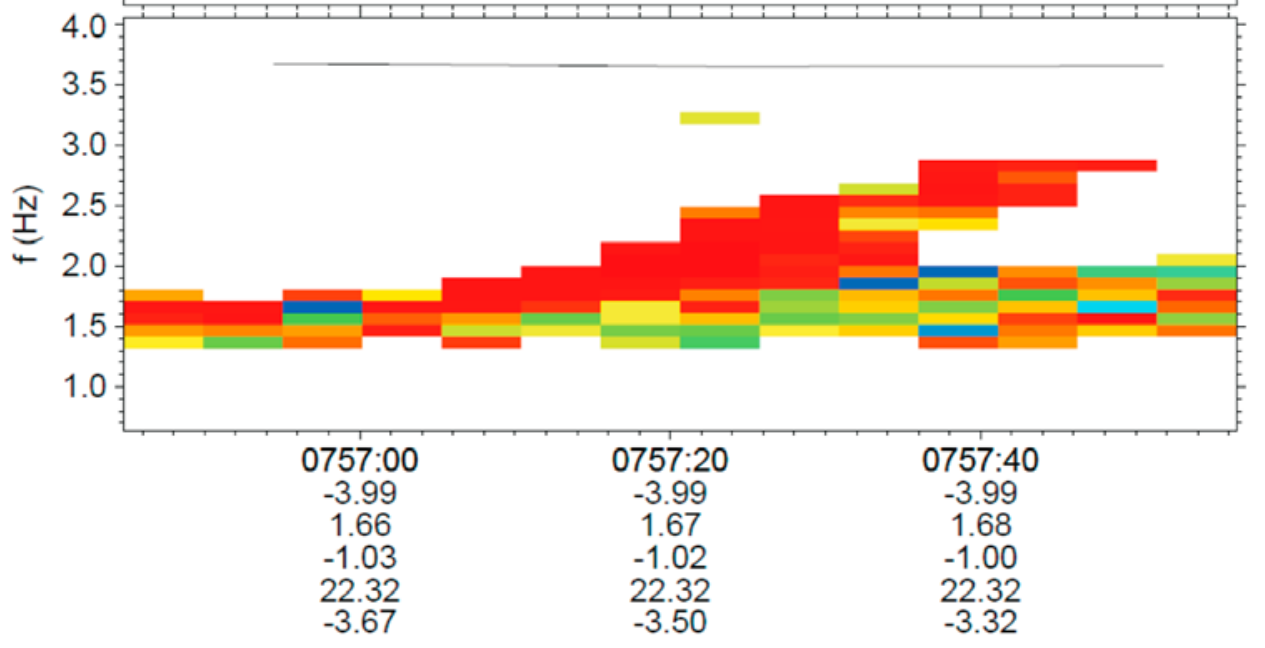

Figure 1. Dynamic power spectra of (a) magnetic, (b) electric field in a frequency range of $0.5-4.0 \mathrm{~Hz}$, observed by Cluster 4 during the time 07:56:44.795-07:57:56.475 on 30 March 2002. (c) Polarization and (d) coherency analyses. The spectra are from the STAFF-SC and EFW instruments and the magnetic field measurements from the FGM instrument. 
The electromagnetic field of the wave should satisfy Maxwell's equations expressed as

$$
\begin{gathered}
i \frac{\partial \vec{E}_{w}}{\partial h}=-\frac{\partial \vec{B}_{w}}{\partial t}, \\
i \frac{\partial \vec{B}_{w}}{\partial h}=\mu_{0} \vec{J},
\end{gathered}
$$

where $\vec{J}$ is the conduction current due to motion of charged particles, and we have neglected the displacement current because we assume the phase velocity, $V_{p}=\omega / k$, is much smaller than the speed of light $c$. We neglect the inertia term of the electron momentum equation, which is expressed as

$$
\vec{E}_{w}-i \vec{V}_{e} B_{0}=0,
$$

where $\vec{V}_{e}$ is the velocity of the cold electron motion supporting the EMIC wave propagation, and $B_{0}$ is the absolute value of the static magnetic field. The electron motion contributes to the transverse wave field as the transverse electron current density $\vec{J}_{e}=-e n_{e} \vec{V}_{e}$, where $e$ and $n_{e}$ are the absolute value of electron charge and the electron density, respectively. From (5), we have

$$
\vec{J}_{e}=-i \frac{\epsilon_{0} \omega_{p e}^{2}}{\Omega_{e}} \vec{E}_{w},
$$

where $\epsilon_{0}, \omega_{p e}$, and $\Omega_{e}$ are the electric permittivity of vacuum, the electron plasma frequency, and the electron cyclotron frequency, respectively.

[11] The momentum equation of ions of species "s" is written as

$$
\frac{\partial \vec{V}_{s}}{\partial t}=\frac{q_{s}}{m_{s}}\left(\vec{E}_{w}-i \vec{V}_{s} B_{0}\right),
$$

where $\vec{V}_{s}, q_{s}$, and $m_{s}$ are the velocity of the motion, charge, and mass of ion species "s". The motion of each ion species contributes to the transverse wave field by a current density of species "s" given by $\vec{J}_{s}=q_{s} n_{s} \vec{V}_{s}$, where $n_{s}$ is the charge density of species "s". From (7), we obtain

$$
\frac{\partial J_{s}}{\partial t} \exp \left(i \psi_{J s}\right)+i\left(\Omega_{s}-\omega\right) \vec{J}_{s}=\epsilon_{0} \omega_{p s}^{2} \vec{E}_{w}
$$

where $\omega_{p s}$ is the plasma frequency of species "s". We assume the amplitudes of electromagnetic fields and current densities of particle species vary slowly compared with the wave phase variation represented by $\omega$ and $k$. Namely, assuming $\left(\partial J_{s} / \partial t\right) \ll\left(\Omega_{s}-\omega\right) J_{s}$, we have

$$
J_{s} \exp \left(i \psi_{J s}\right) \sim-i \frac{\epsilon_{0} \omega_{p s}^{2}}{\Omega_{s}-\omega} E_{w} \exp \left(i \psi_{E}\right),
$$

which results in approximate relations

$$
J_{s} \sim \frac{\epsilon_{0} \omega_{p s}^{2}}{\Omega_{s}-\omega} E_{w}
$$

and

$$
\exp \left(i \psi_{J s}\right) \sim-i \exp \left(i \psi_{E}\right)
$$

Substituting (10) and (11) into the first term of (8), which is much smaller than the other terms, we obtain

$$
-i \frac{\epsilon_{0} \omega_{p s}^{2}}{\Omega_{s}-\omega} \frac{\partial E_{w}}{\partial t} \exp \left(i \psi_{E}\right)+i\left(\Omega_{s}-\omega\right) \vec{J}_{s}=\epsilon_{0} \omega_{p s}^{2} \vec{E}_{w} .
$$

After dividing (12) by $\left(\Omega_{s}-\omega\right)$, we take a sum for all ion species to obtain

$$
-i \sum_{s} \frac{\epsilon_{0} \omega_{p s}^{2}}{\left(\Omega_{s}-\omega\right)^{2}} \frac{\partial E_{w}}{\partial t} \exp \left(i \psi_{E}\right)+i \sum_{s} \vec{J}_{s}=\sum_{s} \frac{\epsilon_{0} \omega_{p s}^{2}}{\Omega_{s}-\omega} \vec{E}_{w} .
$$

[12] We decompose the current density $\vec{J}$ in (4) into three components, $\vec{J}_{e}$ for electrons, $\sum_{s} \vec{J}_{s}$ for non-resonant ions, and $\vec{J}_{R}$ for resonant ions, namely,

$$
\vec{J}_{e}+\sum_{s} \vec{J}_{s}+\vec{J}_{R}=\frac{i}{\mu_{0}} \frac{\partial \vec{B}_{w}}{\partial h} .
$$

From (6), (13), and (14), we obtain

$$
-i \sum_{s} \frac{\epsilon_{0} \omega_{p s}^{2}}{\left(\Omega_{s}-\omega\right)^{2}} \frac{\partial E_{w}}{\partial t} \exp \left(i \psi_{E}\right)-\frac{1}{\mu_{0}} \frac{\partial \vec{B}_{w}}{\partial h}-i \vec{J}_{R}=\epsilon_{0} \Pi_{c} \vec{E}_{w},
$$

where we have introduced an angular frequency $\Pi_{c}$ defined by

$$
\Pi_{c}=\sum_{s} \frac{\omega_{p s}^{2}}{\Omega_{s}-\omega}-\frac{\omega_{p e}^{2}}{\Omega_{e}} .
$$

We decompose the resonant current $\vec{J}_{R}$ into $\vec{J}_{E}$ and $\vec{J}_{B}$ parallel to the wave electric field $\vec{E}_{w}$ and the wave magnetic field $\vec{B}_{w}$, respectively. Noting that $\exp \left(i \psi_{B}\right)=i \exp \left(i \psi_{E}\right)$, we have

$$
\vec{J}_{R}=\left(J_{E}+i J_{B}\right) \exp \left(i \psi_{E}\right) .
$$

We differentiate both sides of (15) neglecting the derivatives of the amplitudes and phases that are higher than the first order. Using (3) and (17), we obtain

$$
\begin{gathered}
-\sum_{s} \frac{\epsilon_{0} \omega \omega_{p s}^{2}}{\left(\Omega_{s}-\omega\right)^{2}} \frac{\partial E_{w}}{\partial t} \exp \left(i \psi_{E}\right)+\frac{i}{\mu_{0}}\left[2 i k \frac{\partial E_{w}}{\partial h} \exp \left(i \psi_{E}\right)-k^{2} \vec{E}_{w}\right] \\
=\epsilon_{0} \Pi_{c}\left[\frac{\partial E_{w}}{\partial t} \exp \left(i \psi_{E}\right)-i \omega \vec{E}_{w}\right]+\omega\left(J_{E}+i J_{B}\right) \exp \left(i \psi_{E}\right) .
\end{gathered}
$$

Multiplying (18) by $\exp \left(-i \psi_{E}\right)$, and separating the real and imaginary parts, we obtain

$$
\epsilon_{0}\left[\Pi_{c}+\omega \sum_{s} \frac{\omega_{p s}^{2}}{\left(\Omega_{s}-\omega\right)^{2}}\right] \frac{\partial E_{w}}{\partial t}+\frac{2 k}{\mu_{0}} \frac{\partial E_{w}}{\partial h}=-\omega J_{E}
$$

and

$$
\left(-\frac{k^{2}}{\mu_{0}}+\omega \epsilon_{0} \Pi_{c}\right) E_{w}=\omega J_{B}
$$


Noting that (3) gives $E_{w}=(\omega / k) B_{w},(19)$ and (20) are rewritten as

$$
\frac{\partial B_{w}}{\partial t}+V_{g} \frac{\partial B_{w}}{\partial h}=-\frac{\mu_{0} V_{g}}{2} J_{E}
$$

and

$$
c^{2} k^{2}-\omega \Pi_{c}=-\mu_{0} c^{2} k \frac{J_{B}}{B_{w}},
$$

where $V_{g}$ is the group velocity given by

$$
V_{g}=2 c^{2} k\left[\Pi_{c}+\omega \sum_{s} \frac{\omega_{p s}^{2}}{\left(\Omega_{s}-\omega\right)^{2}}\right]^{-1} .
$$

The wave equations (21) and (22) take essentially the same forms as the wave equations for a whistler-mode wave [Omura and Matsumoto, 1982; Omura et al., 2009]. A generalized derivation of the wave equations for whistler and Alfven mode cyclotron masers is found by Trakhtengerts and Rycroft [2008].

[13] We evaluate the magnitude of the resonant currents $J_{E}$ and $J_{B}$ in terms of the growth rate $\omega_{i}$. Neglecting the convective term in (21), we have

$$
\omega_{i} B_{w}=-\frac{\mu_{0}}{2} V_{g} J_{E} .
$$

Since $\vec{J}_{E}$ and $\vec{J}_{B}$ are projections of the resonant current $\vec{J}_{R}$, we can assume $\left|\vec{J}_{E}\right| \sim\left|\vec{J}_{B}\right|$. From (24), we obtain

$$
\frac{J_{B}}{B_{w}} \sim \pm \frac{2 \omega_{i}}{\mu_{0} V_{g}}
$$

Using (25), we rewrite the nonlinear dispersion relation given by (22) as

$$
c^{2} k^{2}\left(1 \pm 2 \frac{V_{p}}{V_{g}} \frac{\omega_{i}}{\omega}\right)=\omega \Pi_{c}
$$

Assuming $\omega_{i} \ll \omega$ and $V_{p} \sim V_{g}$, we find that (26) is reduced to the cold plasma dispersion relation

$$
c^{2} k^{2}=\omega \Pi_{c}
$$

which gives an expression of the phase velocity as a function of $\omega$,

$$
V_{p}=c \sqrt{\frac{\omega}{\Pi_{c}}}
$$

Differentiating (27) with respect to $t$, and noting that $\partial k / \partial t=$ $-\partial \omega / \partial h$, we obtain

$$
\frac{\partial \omega}{\partial t}+V_{g} \frac{\partial \omega}{\partial h}=0
$$

Along with (21), (29) describes a wave packet of an EMIC wave propagating at the group velocity $V_{g}$ with a constant wave frequency $\omega$ and an evolving wave amplitude $B_{w}$ due to the resonant current $J_{E}$. This indicates that the essential physical process of the frequency variation takes place only at the source of the emissions near the magnetic equator as analyzed in the following sections.

\section{Nonlinear Dynamics of Resonant Protons}

[14] We study the dynamics of energetic protons interacting with an EMIC wave through cyclotron resonance. We calculate partial derivatives of variables in preparation for the calculation of the second-order cyclotron resonance condition. Differentiating (27) with respect to $h$, we obtain

$$
2 c^{2} k \frac{\partial k}{\partial h}=\frac{\partial \omega}{\partial h} \Pi_{c}+\omega \frac{\partial \Pi_{c}}{\partial h} .
$$

We assume that the densities of ions and electrons vary proportionally with the intensity of the ambient magnetic field, i.e., $\omega_{p s}^{2} \propto \Omega_{s}$ and $\omega_{p e}^{2} \propto \Omega_{e}$. From (16), we obtain

$$
\frac{\partial \Pi_{c}}{\partial h}=\sum_{s}\left[\frac{\omega_{p s}^{2}}{\left(\Omega_{s}-\omega\right)^{2}}\left(\frac{\partial \omega}{\partial h}-\frac{\omega}{\Omega_{s}} \frac{\partial \Omega_{s}}{\partial h}\right)\right] .
$$

Substituting (31) into (30), and using (23) and (29), we obtain

$$
\frac{\partial k}{\partial h}=-\frac{1}{V_{g}^{2}} \frac{\partial \omega}{\partial t}+\frac{\omega}{\Omega_{H}}\left(\frac{\Pi_{c}}{2 c^{2} k}-\frac{1}{V_{g}}\right) \frac{\partial \Omega_{H}}{\partial h} .
$$

From the cyclotron resonance condition the resonance velocity is given by

$$
V_{R}=\frac{\omega-\Omega_{H}}{k} .
$$

Using (32), we calculate time variation of $V_{R}$ seen by a proton moving with a parallel velocity $v_{\|}$as

$$
\begin{aligned}
\frac{\mathrm{d} V_{R}}{\mathrm{~d} t}= & \frac{1}{k}\left(1-\frac{v_{\|}}{V_{g}}\right)\left(1-\frac{V_{R}}{V_{g}}\right) \frac{\partial \omega}{\partial t} \\
& -\frac{v_{\|}}{k}\left[1+\frac{\omega V_{R}}{\Omega_{H}}\left(\frac{\Pi_{c}}{2 c^{2} k}-\frac{1}{V_{g}}\right)\right] \frac{\partial \Omega_{H}}{\partial h} .
\end{aligned}
$$

[15] We define a relative phase $\zeta$ between the phase $\phi$ of the perpendicular velocity $\vec{V}_{\perp 0}$ of a proton and the phase $\psi_{B}$ of the wave magnetic field $\vec{B}_{w}$. Noting that the proton undergoes cyclotron motion with a cyclotron radius $r_{c}=V_{\perp 0} / \Omega_{H}$ and accelerated by the mirror force due to the radial component of the nonuniform magnetic field $B_{r}=-\left(r_{c} / 2\right) \partial B_{0} / \partial h$, we can write the equation of motion as

$$
\frac{\mathrm{d} v_{\|}}{\mathrm{d} t}=-\Omega_{w} V_{\perp 0} \sin \zeta-\frac{V_{\perp 0}^{2}}{2 \Omega_{H}} \frac{\partial \Omega_{H}}{\partial h},
$$

where $\Omega_{w}=e B_{w} / m_{H}$.

[16] We calculate the first-order phase variation

$$
\frac{\mathrm{d} \zeta}{\mathrm{d} t}=\frac{\mathrm{d} \phi}{\mathrm{d} t}-\frac{\mathrm{d} \psi_{B}}{\mathrm{~d} t}=-\Omega_{H}-\left(k v_{\|}-\omega\right) .
$$

Using the resonance velocity $V_{R}$ defined by (33), we have

$$
\frac{\mathrm{d} \zeta}{\mathrm{d} t}=-k\left(v_{\|}-V_{R}\right)
$$




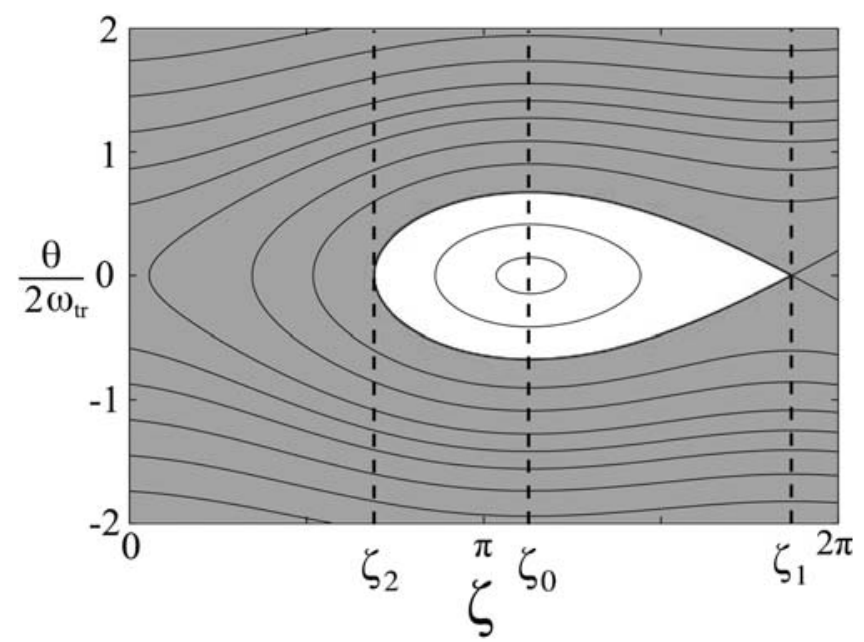

Figure 2. Trajectories of resonant protons in the $(\theta-\zeta)$ phase space for the inhomogeneity ratio $S=0.4$. The phase angle $\zeta_{0}$ is the center of trapping motion, while $\zeta_{1}$ is the saddle point and $\zeta_{2}$ is the boundary of the trapping region at $\theta=0$.

Assuming $v_{\|} \sim V_{R}$, we calculate the second-order phase variation from (37)

$$
\frac{\mathrm{d}^{2} \zeta}{\mathrm{d} t^{2}}=-k \frac{\mathrm{d}}{\mathrm{d} t}\left(v_{\|}-V_{R}\right)
$$

Using (34) and (35), we obtain the second-order nonlinear ordinary differential equation for the phase angle $\zeta$,

$$
\frac{\mathrm{d}^{2} \zeta}{\mathrm{d} t^{2}}=\omega_{t r}^{2}(\sin \zeta+S)
$$

where $\omega_{t r}$ is the trapping frequency given by $\omega_{t r}=\sqrt{k V_{\perp 0} \Omega_{w}}$. The parameter $S$ is the inhomogeneity ratio given by

$$
S=\frac{1}{s_{0} \omega \Omega_{w}}\left(s_{1} \frac{\partial \omega}{\partial t}+V_{p} s_{2} \frac{\partial \Omega_{H}}{\partial h}\right)
$$

where

$$
\begin{gathered}
s_{0}=\frac{V_{\perp 0}}{V_{p}}, \\
s_{1}=\left(1-\frac{V_{R}}{V_{g}}\right)^{2}, \\
s_{2}=\left(\frac{V_{\perp 0}^{2}}{2 V_{p}^{2}}+\frac{V_{R}^{2}}{V_{p} V_{g}}-\frac{V_{R}^{2}}{2 V_{p}^{2}}\right) \frac{\omega}{\Omega_{H}}-\frac{V_{R}}{V_{p}} .
\end{gathered}
$$

If we assume that the densities of cold ions and electrons are constant along the magnetic field line in the derivation above, the third term in the brackets of (43) disappears.
[17] If $|S|<1$, the second-order resonance condition

$$
\frac{\mathrm{d}^{2} \zeta}{\mathrm{d} t^{2}}=0
$$

is satisfied by a resonant proton with $v_{\|}=V_{R}$ and $\zeta=-\sin ^{-1} S$.

\section{Nonlinear Wave Growth}

[18] We assume the parabolic variation of the dipole magnetic field near the equator expressed as $\Omega_{H}=\Omega_{H 0}(1+$ $\left.a h^{2}\right)$, where $a=4.5 /\left(L R_{E}\right)^{2}$, corresponding to the Earth's approximate dipole magnetic field. Noting that $\partial \Omega_{H} / \partial h=$ $2 a \Omega_{H 0} h$, we consider a distance $h_{c}$ at which the first and second terms of the r.h.s. of (40) become equal. Equating the two terms, we obtain the critical distance $h_{c}$ as

$$
h_{c}=\frac{1}{2 a V_{p} \Omega_{H 0}}\left(\frac{s_{1}}{s_{2}}\right) \frac{\partial \omega}{\partial t} .
$$

The distance $h_{c}$ is used in identifying the dominant terms of the inhomogeneity ratio $S$ in the following sections.

[19] From the analysis of trajectories of resonant ions as described by the second-order equation, it is found that the maximum value of $J_{E}$ is realized when $|S|=0.4$ [Omura et $a l ., 2008]$. The magnitude of $J_{E}$ is calculated by assuming a velocity distribution function in the velocity phase space in the presence of a coherent EMIC wave as

$$
g\left(v_{\|}, \zeta\right)=g_{0}\left(v_{\|}\right)-Q g_{t r}\left(v_{\|}, \zeta\right)
$$

and we have

$$
J_{E}=e Q V_{\perp 0}^{2} \int_{0}^{2 \pi} \int_{-\infty}^{\infty} g_{t r}\left(v_{\|}, \zeta\right) \sin \zeta \mathrm{d} v_{\|} \mathrm{d} \zeta
$$

where we have assumed a delta function for the perpendicular velocity at $v_{\perp}=V_{\perp 0}$. The functions $g_{0}\left(v_{\|}\right)$and $g_{t r}\left(v_{\|}, \zeta\right)$ are the unperturbed velocity distribution function, and the part of $g_{0}$ that is trapped by the wave, respectively. Since the separatrix of the trapping wave potential is closed, entrapping of new particles does not take place unless the wave amplitude increases. Therefore, there arises an electromagnetic proton hole in the velocity phase space as shown in Figure 2, where we have introduced the new variable $\theta=$ $k\left(v_{\|}-V_{R}\right)$. We assume a factor $Q$ that represents the depth of the proton hole. If $Q=1$ the proton hole is completely void. If $50 \%$ of trapped ions are lost from the trapping wave potential, $Q=0.5$. Assuming that $g_{t r}\left(v_{\|}, \zeta\right)=$ $G$ (constant) inside the trapping region and $g_{t r}\left(v_{\|}, \zeta\right)=0$ outside it, we rewrite (47) as

$$
J_{E}=J_{0} \int_{\zeta_{2}}^{\zeta_{1}}\left[\cos \zeta_{1}-\cos \zeta+S\left(\zeta-\zeta_{1}\right)\right]^{1 / 2} \sin \zeta \mathrm{d} \zeta
$$

where $J_{0}=(2 e)^{3 / 2}\left(m_{H} k\right)^{-1 / 2} V_{\perp 0}^{5 / 2} Q G B_{w}^{1 / 2}$, and $e$ and $m_{H}$ are the charge and rest mass of a proton. The phase angles $\zeta_{1}$ and $\zeta_{2}$ are the boundary of the trapping wave potential as defined in Figure 2. The current $J_{E}$ is a function of $S$ and it 
maximizes at $\mathrm{S}=0.4$. The maximum value is $J_{E} / J_{0}=$ $0.975 \sim 1$. We thus have

$$
J_{E, \max }=(2 e)^{3 / 2}\left(m_{H} k\right)^{-1 / 2} V_{\perp 0}^{5 / 2} B_{w}^{1 / 2} Q G .
$$

[20] Writing the right-hand side of (21) as $\mathrm{d} B_{w} / \mathrm{d} t$ we obtain

$$
\frac{\mathrm{d} B_{w}}{\mathrm{~d} t}=\frac{\mu_{0} V_{g}}{2}(2 e)^{3 / 2}\left(\frac{V_{p}}{m_{H} \omega}\right)^{1 / 2} V_{\perp 0}^{5 / 2} B_{w}^{1 / 2} Q G
$$

where we have eliminated the wave number $k$ using (28). We assume that the velocity distribution function of hot energetic protons is given in terms of the velocities parallel and perpendicular to the ambient magnetic field as

$$
f\left(v_{\|}, v_{\perp}\right)=\frac{n_{h}}{(2 \pi)^{3 / 2} V_{t \|} V_{\perp 0}} \exp \left(-\frac{v_{\|}^{2}}{2 V_{t \|}^{2}}\right) \Delta\left(v_{\perp}-V_{\perp 0}\right)
$$

where $\Delta\left(v_{\perp}\right)$ is the Dirac delta function. The distribution function $f\left(v_{\|}, v_{\perp}\right)$ is normalized to the density of hot protons $n_{h}$. Integrating over $v_{\perp}$, we obtain the magnitude $G$ of the unperturbed distribution function $g\left(v_{\|}, \zeta\right)$ averaged over $v_{\perp}$ at the resonance velocity $V_{R}$,

$$
G=\frac{n_{h}}{(2 \pi)^{3 / 2} V_{t \|} V_{\perp 0}} \exp \left(-\frac{V_{R}^{2}}{2 V_{t \|}^{2}}\right) .
$$

Combining (50) and (52), we obtain

$$
\frac{\mathrm{d} B_{w}}{\mathrm{~d} t}=\Gamma_{N L} B_{w},
$$

where

$$
\Gamma_{N L}=\omega_{p h}^{2} \frac{Q}{2}\left(\frac{V_{p}}{c \Omega_{w} \omega}\right)^{1 / 2} \frac{V_{g}}{V_{t \|}}\left(\frac{V_{\perp 0}}{c \pi}\right)^{3 / 2} \exp \left(-\frac{V_{R}^{2}}{2 V_{t \|}^{2}}\right)
$$

and where we have normalized the wave magnetic field in terms of the proton cyclotron frequency by defining $\Omega_{w}=$ $e B_{w} / m_{H}$, and $\omega_{p h}$ is the plasma frequency of hot protons defined by $\omega_{p h}^{2}=n_{h} e^{2} /\left(m_{H} \epsilon_{0}\right)$. It is noted that $\Gamma_{N L}$ is the nonlinear growth rate [Omura et al., 2009] defined in analogy with the linear growth rate.

\section{Self-Sustaining Rising Tone Emission}

[21] We derive a necessary condition for an EMIC triggered rising tone emission to be amplified through propagation from the equator to the higher latitude region. Separating the derivative of (53) into temporal and spatial derivatives, we obtain

$$
\frac{\partial B_{w}}{\partial t}+V_{g} \frac{\partial B_{w}}{\partial h}=\Gamma_{N L} B_{w}
$$

For the triggered emission to grow at the equator, the temporal growth rate should be positive, i.e., $\partial B_{w} / \partial t>0$. From (55) we obtain

$$
\frac{\partial \Omega_{w}}{\partial h}<\frac{\Gamma_{N L}}{V_{g}} \Omega_{w}
$$

where we assume that EMIC waves propagate in the positive direction, i.e., $V_{g}>0$.

[22] We have found that whistler-mode chorus elements with rising tones are generated at the equator [Katoh and Omura, 2007; Omura et al., 2008]. The linear growth rate of the L-mode EMIC wave instability also maximizes at the equator because the absolute value of the resonance velocity takes the lowest value, and thus the flux of the resonant protons maximizes at the equator. Therefore, the wave amplitude grows fastest, reaching the threshold value for the nonlinear wave growth at the equator.

[23] At the equator the inhomogeneity of the magnetic field is zero, and the second term on the r.h.s. of (40) vanishes. Since the maximum nonlinear wave growth takes place with $S=0.4$, we have the relation between the frequency sweep rate and the wave amplitude at the equator $\Omega_{w 0}$ from (40)

$$
\frac{\partial \omega}{\partial t}=\frac{0.4 s_{0} \omega}{s_{1}} \Omega_{w 0}
$$

As indicated by (29), the frequency does not change in the frame of reference moving with the group velocity $V_{g}$.

[24] As the triggered emission propagates significantly away from the equator to the distance $h\left(\gg h_{c}\right)$, the second term of the inhomogeneity ratio (40) becomes much greater than the first term. For the triggered emission to keep the maximum growth at this distance, the negative resonant current $J_{E}$ must be formed with $S=0.4$. Neglecting the first term on the r.h.s. of (40) and setting $S=0.4$, we obtain

$$
\Omega_{w}=\frac{V_{p} s_{2}}{0.4 s_{0} \omega} \frac{\partial \Omega_{H}}{\partial h} .
$$

Taking the spatial derivative, we obtain

$$
\frac{\partial \Omega_{w}}{\partial h}=\frac{V_{p} s_{2}}{0.4 s_{0} \omega} \frac{\partial^{2} \Omega_{H}}{\partial h^{2}}=\frac{5 V_{p} a s_{2} \Omega_{H 0}}{s_{0} \omega} .
$$

Since the spatial gradient of the wave amplitude $\partial \Omega_{w} / \partial h$ is a constant in the parabolic magnetic field, formation of the constant spatial gradient of the wave amplitude from the generation point near the magnetic equator to the spatial limit of the maximum wave growth is a sufficient condition for the self-sustaining nonlinear wave growth. The spatial gradient does not depend on the wave amplitude itself. When the optimum self-sustaining wave growth is realized, the gradient of the wave amplitude should be close to the value given by (59).

[25] Inserting (59) into (56), we obtain

$$
\Omega_{w}>\frac{5 V_{p} a s_{2} \Omega_{H 0} V_{g}}{s_{0} \omega \Gamma_{N L}} .
$$




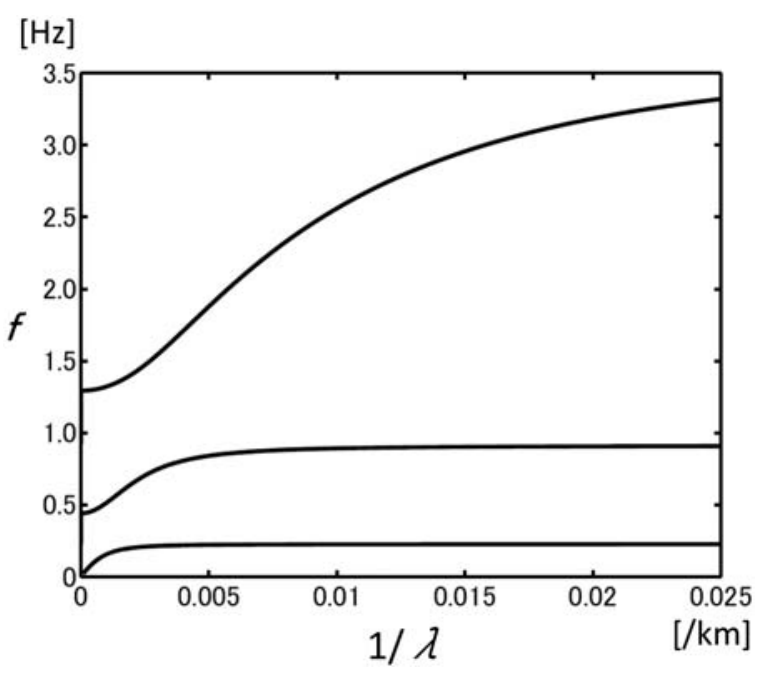

Figure 3. Dispersion relation for L-mode EMIC waves with frequency $\omega /(2 \pi)=f$ and wave number $k /(2 \pi)=1 / \lambda$.

Using the normalized parameters, $\tilde{V}_{\perp 0}=V_{\perp Q} / c, \tilde{\omega}=\omega / \Omega_{H 0}$, $\tilde{a}=a c^{2} / \Omega_{H 0}^{2}$, and likewise for $\tilde{V}_{t \|}, \tilde{\omega}_{p h}$, and $\Omega_{w 0}$, we rewrite $(60)$ as

$$
\tilde{\Omega}_{w 0}=\frac{B_{w}}{B_{0}}>\tilde{\Omega}_{t h}
$$

where

$$
\tilde{\Omega}_{t h}=\frac{100 \pi^{3} \tilde{V}_{p}^{3}}{\tilde{\omega} \tilde{\omega}_{p h}^{4} \tilde{V}_{\perp 0}^{5}}\left(\frac{\tilde{a} s_{2} \tilde{V}_{t \|}}{Q}\right)^{2} \exp \left(\frac{\tilde{V}_{R}^{2}}{\tilde{V}_{t \|}^{2}}\right)
$$

[26] As is obvious from (58), the self-sustaining mechanism only works for $h>0$. Namely the nonlinear wave growth takes place when the wave propagates away from the equator with an amplitude satisfying (61).

[27] The nonlinear wave growth is due to the formation of a resonant current as described by the second-order resonance condition, while the linear wave growth is caused by particle motion along the diffusion curve at the resonance velocity defined by the first-order resonance condition. The Poynting vector analysis for the EMIC waves [Pickett et al., 2010] indicates that the triggering EMIC wave at $1.5 \mathrm{~Hz}$ seems to show some positive divergence in the equatorial region. The triggering EMIC wave is likely to be excited near the equator due to the linear growth driven by the temperature anisotropy. As shown by the linear dispersion analysis in the following section, the triggering wave at $1.5 \mathrm{~Hz}$ is close to the cut-off frequency at $1.3 \mathrm{~Hz}$ (see Figure 3). The spatial linear growth rate takes the maximum value near the wave cut-off frequency [Gendrin et al., 1984]. The coherent component of the triggering wave forms an electromagnetic proton hole in the velocity phase space. Once the amplitude of the coherent wave exceeds the threshold value for self-sustaining emissions, nonlinear wave growth sets in driven by the second-order phase variation $\partial \omega / \partial t$ corresponding to the maximum value of the resonant current $J_{E}$.
[28] We evaluate the temporal variation of the wave amplitude by assuming that the spatial derivative of the wave amplitude in (55) takes the threshold value for the selfsustaining wave growth given by (59). Assuming the minimum spatial gradient of the growing wave amplitude in (59), and inserting into (55), we obtain

$\frac{\partial \tilde{\Omega}_{w 0}}{\partial \tilde{t}}=\tilde{V}_{g}\left[\frac{Q \tilde{\omega}_{p h}^{2}}{2 \tilde{V}_{t \|}}\left(\frac{\tilde{V}_{\perp 0}}{\pi}\right)^{3 / 2}\left(\frac{\tilde{V}_{p} \tilde{\Omega}_{w 0}}{\tilde{\omega}}\right)^{1 / 2} \exp \left(-\frac{\tilde{V}_{R}^{2}}{2 \tilde{V}_{t \|}^{2}}\right)-\frac{5 \tilde{V}_{p} s_{2} \tilde{a}}{s_{0} \tilde{\omega}}\right]$.

Rewriting (57) we obtain

$$
\frac{\partial \tilde{\omega}}{\partial \tilde{t}}=\frac{2 s_{0}}{5 s_{1}} \tilde{\omega} \tilde{\Omega}_{w 0}
$$

The temporal evolution of an EMIC triggered emission at the equator is determined by a set of equations (63) and (64). Since these equations are essentially the same as the chorus equations derived for whistler-mode chorus emissions [Omura et al., 2009], we call them the EMIC chorus equations in the following.

\section{Numerical Solutions of EMIC Chorus Equations and Comparison With Observations}

[29] We first determine the linear dispersion relation for the EMIC triggered emission event observed by the Cluster spacecraft on 30 March 2002 as shown in Figure 1. In the absence of the measurement of the low energy thermal ions, we make use of the cut-off frequencies of the L-mode EMIC wave dispersion. The cut-off frequency is obtained by assuming $k=0$ in (27). The spacecraft identified three distinct ion species, $\mathrm{H}^{+}, \mathrm{He}^{+}$and $\mathrm{O}^{+}$. Writing $\Pi_{c}=0$ explicitly, we obtain

$$
\frac{\omega_{p H}^{2}}{\Omega_{H}-\omega}+\frac{\omega_{p H e}^{2}}{\Omega_{H e}-\omega}+\frac{\omega_{p O}^{2}}{\Omega_{O}-\omega}=\frac{\omega_{p e}^{2}}{\Omega_{e}},
$$

where $\left(\omega_{p H}, \omega_{p H e}, \omega_{p O}\right)$ and $\left(\Omega_{H}, \Omega_{H e}, \Omega_{O}\right)$ are the plasma frequencies and cyclotron frequencies of the $\mathrm{H}^{+}, \mathrm{He}^{+}$, and $\mathrm{O}^{+}$ions, respectively. Normalizing the densities of proton, helium, and oxygen ions by the electron density as $\tilde{n}_{H}=n_{H} / n_{e}$, $\tilde{n}_{H e}=n_{H e} / n_{e}, \tilde{n}_{O}=n_{O} / n_{e}$, we obtain from (65)

$$
\frac{\tilde{n}_{H}}{1-\tilde{\omega}}+\frac{\tilde{n}_{H e}}{1-4 \tilde{\omega}}+\frac{\tilde{n}_{O}}{1-16 \tilde{\omega}}=1
$$

where we use the relations $\Omega_{H e}=\Omega_{H} / 4$ and $\Omega_{O}=\Omega_{H} / 16$. The charge neutrality condition gives

$$
\tilde{n}_{H}+\tilde{n}_{H e}+\tilde{n}_{O}=1 .
$$

[30] The frequency spectra shown in Figure 1 indicate that the cut-off frequency of the upper L-mode EMIC mode bounded by the proton cyclotron frequency is $\sim 1.3 \mathrm{~Hz}$. The magnetic field measurement indicates that the proton cyclotron frequency is $\sim 3.7 \mathrm{~Hz}$. The particle measurement indicates that the densities of helium and oxygen ions are nearly equal. Setting $\tilde{\omega}=1.3 / 3.7$ and $\tilde{n}_{H e}=\tilde{n}_{O}$ in (66) and (67), we obtain $\tilde{n}_{H}=0.81$ and $\tilde{n}_{H e}=\tilde{n}_{O}=0.095$. The 


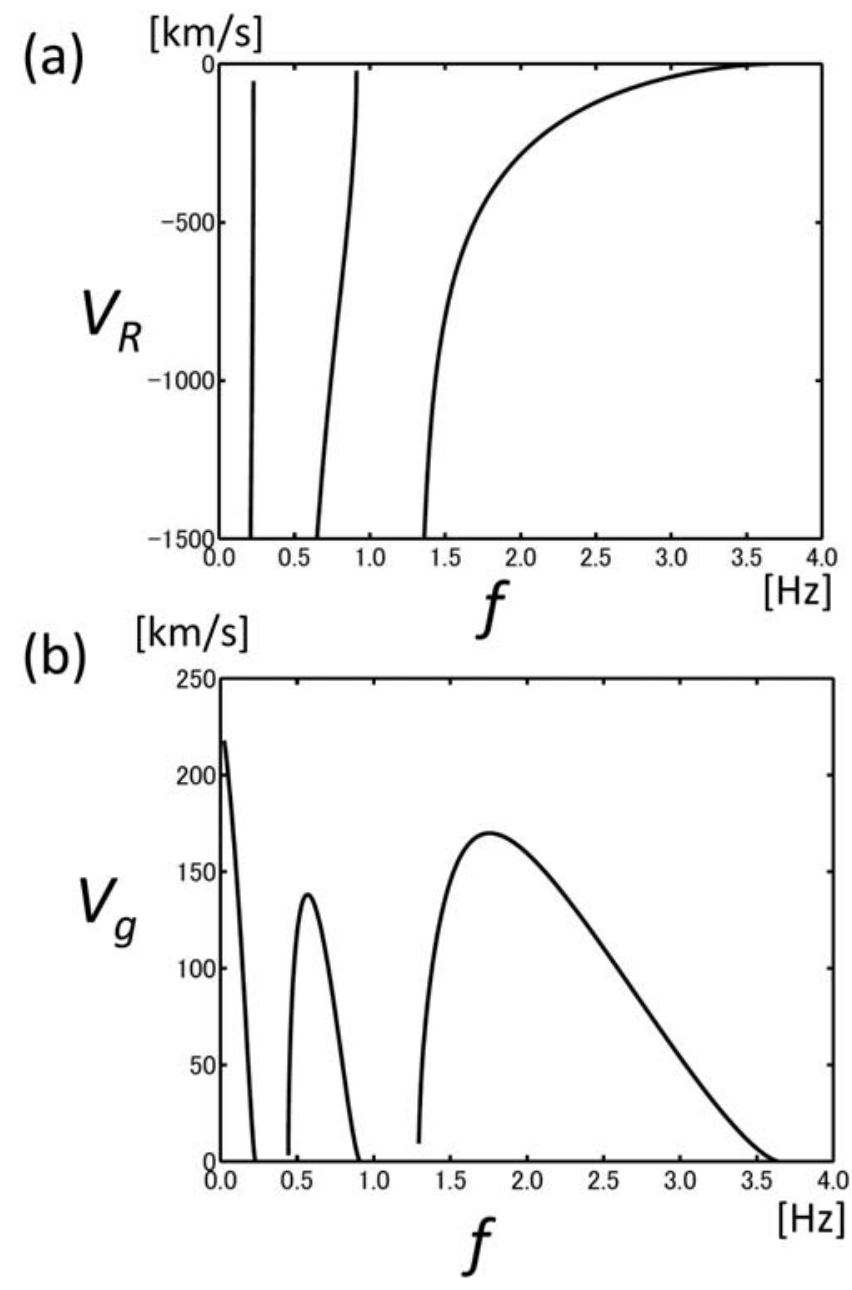

Figure 4. (a) The resonance velocity $V_{R}$ and (b) the group velocity $V_{g}$ of L-mode EMIC waves as functions of a frequency $f$.

WHISPER and EFW instruments of the Cluster spacecraft can determine the electron density as $n_{e} \sim 178 / \mathrm{cc}$, and thus we can estimate that $n_{H}=144 / \mathrm{cc}, n_{H e}=17 / \mathrm{cc}$, and $n_{O}=17 / \mathrm{cc}$. We plot the dispersion relation of the L-mode EMIC waves in Figure 3. As the frequency $f$ increases from $1.5 \mathrm{~Hz}$ to $3.0 \mathrm{~Hz}$, the wavelength $\lambda$ decreases from $370 \mathrm{~km}$ to $63 \mathrm{~km}$. In Figure 4 , we plot the cyclotron resonance velocity $V_{R}$ given by (33) and the group velocity $V_{g}$ given by (23). It is noted that the group velocity is $\sim 150 \mathrm{~km} / \mathrm{s}$, and it is the velocity of the wave packet generated around the magnetic equator. The resonance velocity varies as $-800 \mathrm{~km} / \mathrm{s}$ for $f=$ $1.5 \mathrm{~Hz},-500 \mathrm{~km} / \mathrm{s}$ for $f=1.7 \mathrm{~Hz}$, and $-120 \mathrm{~km} / \mathrm{s}$ for $f=$ $2.5 \mathrm{~Hz}$. The ratio of $V_{R} / V_{g}$ appears in (42) and controls the frequency sweep rate (64).

[31] Figure 5 shows the cross sections of the measured velocity distribution functions at different times during the EMIC triggered emission event. Figure 5 (top) shows enhanced temperature anisotropies in the velocity range below $1000 \mathrm{~km} / \mathrm{s}$. We can find enhanced proton flux around $v_{\perp}=800 \mathrm{~km} / \mathrm{s}$. Therefore, we assume $V_{\perp 0}=800 \mathrm{~km} / \mathrm{s}$ and $V_{t \|}=600 \mathrm{~km} / \mathrm{s}$ so that the unperturbed velocity distribution function has some temperature anisotropy that can excite the EMIC waves with a positive linear growth rate.

[32] We solve equations (63) and (64) numerically for the realistic parameters observed by Cluster 4 at $L=4.27$. Based on the analysis presented above, we assumed $\omega_{p H}=679 \Omega_{H 0}$, $\omega_{p O}=58.3 \Omega_{H 0}, \omega_{p H e}=117 \Omega_{H 0}, V_{t \|}=0.002 c, V_{\perp 0}=$ $0.00267 c$, and $Q=0.5$. We integrate the wave amplitude $B_{w}$ and the frequency $\omega$ from $\omega=0.4 \Omega_{H 0}$ starting with the observed initial wave amplitude of $0.5 \mathrm{nT}$. We tried numerical integration with different values of the energetic proton density $n_{h}$ that forms the distribution function assumed in (51). With $n_{h} / n_{H}=0.05$, we find a solution that grows in time with the longest time duration, while the wave damps out in time with a lower density. With the same density $n_{h} / n_{H}=0.05$, we varied the initial wave amplitude as shown in Figure 6 . The time duration of the emission becomes longest when the wave amplitude is just above the threshold wave amplitude, which is computed as $0.48 \mathrm{nT}$ from (62). Once the wave amplitude $B_{w}$ exceeds the threshold, it increases rapidly because of the strong nonlinear wave growth mechanism.

[33] We have assumed that a wave with a positive phase velocity interacts with resonant protons with negative parallel velocities. As the wave amplitude increases, the trapping velocity $V_{t r}$, which is the width of the electromagnetic proton hole [Omura et al., 2008], also increases. As the frequency increases, the absolute value of the resonance velocity decreases. If the velocity range of the proton hole $\left(v_{\|}=V_{R} \pm V_{t r}\right)$ overlaps with that of the cold protons $\left(v_{\|}=0\right)$ supporting the wave propagation, the motion of the cold protons becomes highly nonlinear, invalidating the linear dispersion relation assumed in the present theory. Therefore, the following condition has to be satisfied as an extreme limit of the nonlinear wave growth:

$$
V_{R}+V_{t r}<0
$$

where

$$
V_{t r}=\frac{2 \omega_{t r}}{k}=2 \sqrt{\frac{V_{\perp 0} \Omega_{w 0}}{k}}
$$

The condition is written for the normalized wave amplitude as

$$
\tilde{\Omega}_{w 0} \leq \frac{\tilde{V}_{p}}{4 \tilde{V}_{\perp 0}} \frac{(1-\tilde{\omega})^{2}}{\tilde{\omega}}
$$

[34] We have followed the time evolution of the wave amplitude $\Omega_{w 0}$ by integrating (55) as far as the inequality (70) is satisfied. Once the inequality is violated by the growing wave amplitude, we regard the wave amplitude as saturated. The frequency evolution is followed by integrating (64) even after the saturation of the wave amplitude as shown in Figure 6. Because of the large amplitude $\sim 10 \mathrm{nT}$ that gives a large frequency sweep rate as indicated by (64), the time scale of the rising tones is much shorter than the observations.

[35] The detailed analysis of the wave amplitude and phase shown in Figure 7 indicates that there occurs a saturation of the wave amplitude at $\sim 2.5 \mathrm{nT}$. In integrating the 


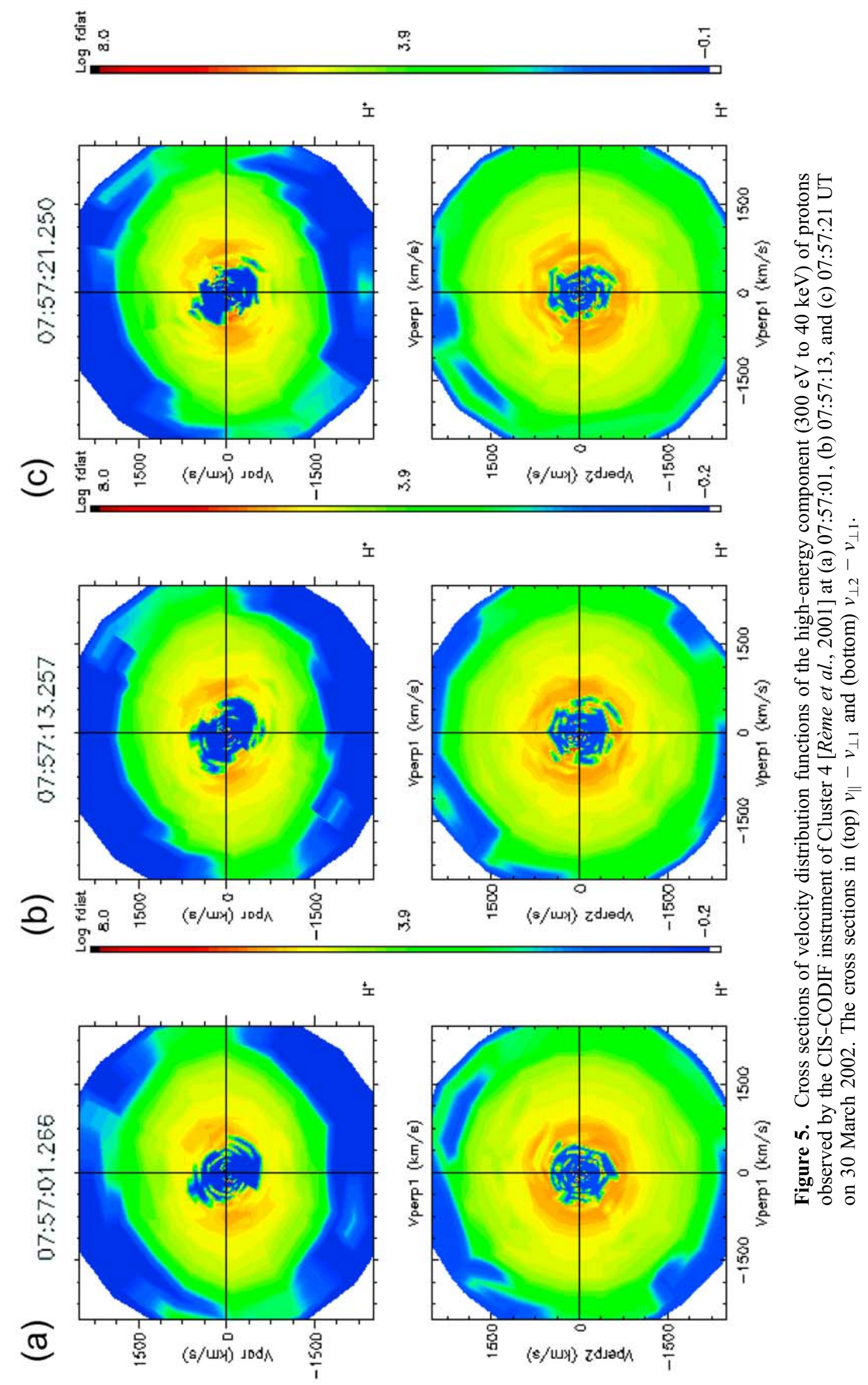


(a) $[\mathrm{nT}]$

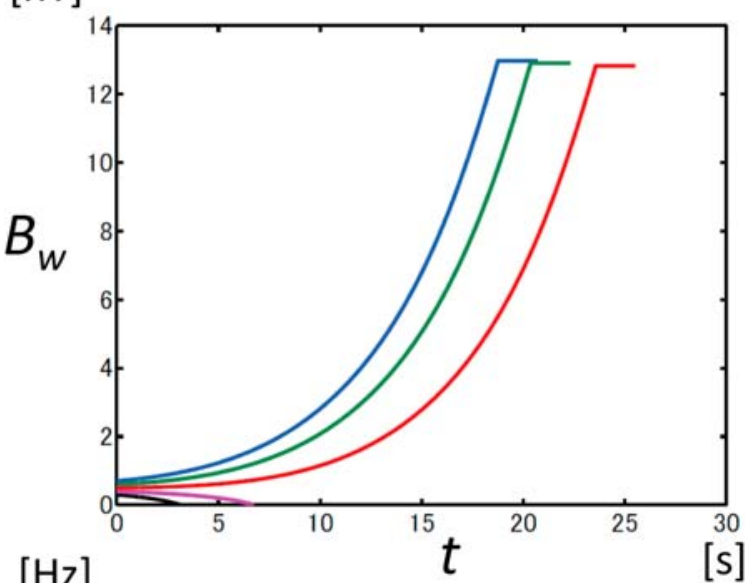

(b) $[\mathrm{Hz}]$

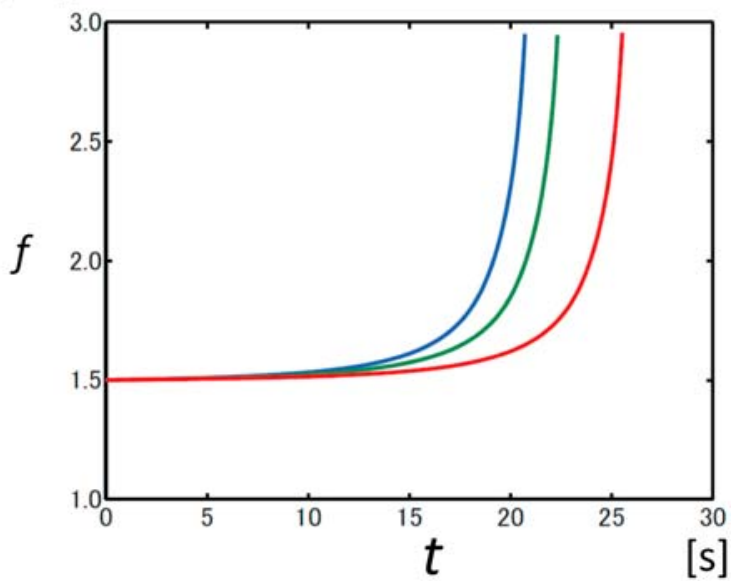

Figure 6. Solutions of the EMIC chorus equations for different initial wave amplitudes indicated by different colors: 0.3 (black), 0.4 (magenta), 0.5 (red), 0.6 (green), and 0.7 (blue) nT. (a) Wave amplitudes and (b) frequencies.

wave amplitude based on (63), we stop the integration once the amplitude exceeds the saturation level of $2.5 \mathrm{nT}$ to model the observed saturation. We integrate the frequency $\omega$ further by using the saturated wave amplitude up to the maximum wave frequency $\sim 3 \mathrm{~Hz}$ observed in the wave spectrum. The results with different initial wave amplitudes are shown in Figure 8. The time scale of the emission is increased to about 40 seconds, which agrees well with the observed rising tone emission shown in Figures 1 and 7. The solutions plotted in Figure 8 show that the frequency sweep rate increases as the frequency increases, while it slightly decreases at higher frequencies in the observation shown in Figure 1. This is due to the variation of the group velocity shown in Figure 4b. The observed triggered emission should have propagated over some distance from the generation point near the magnetic equator. The smaller group velocity at the higher frequency makes the frequency sweep rate smaller.

[36] Using (64), we rewrite (45) as

$$
\tilde{h}_{c}=\frac{s_{0} \tilde{\omega} \tilde{\Omega}_{w 0}}{5 s_{2} \tilde{a} \tilde{V}_{p}} .
$$

In the present case, $h_{c}=320 \mathrm{~km}$ for the initial wave amplitude $0.5 \mathrm{nT}$ and the wave frequency $1.5 \mathrm{~Hz}$. With the saturated wave amplitude $2.5 \mathrm{nT}$ and the wave frequency $3.0 \mathrm{~Hz}, h_{c}=3700 \mathrm{~km}$. Therefore, the nonlinear wave growth driven by the rising frequency takes place over quite a large range of distance from the magnetic equator. Even beyond $h_{c}$, triggered wave packets can grow due to the gradient of the magnetic field as far as there exists a sufficient flux of resonant protons along the magnetic field line.

\section{Summary and Discussion}

[37] We have investigated the nonlinear wave growth mechanism of EMIC rising tone emissions triggered by Pc1 waves. Figure $1 \mathrm{c}$ shows that the Pc1 waves at $1.5 \mathrm{~Hz}$ consist of either right or left handed polarization. This is typical of the Pc1 waves observed during 07:55-08:20 UT on 30 March 2002 [Pickett et al., 2010]. In Figures 1a and 1b, we find a decrease of the Pc1 wave amplitude at $1.5 \mathrm{~Hz}$ by one order of magnitude just before 07:57. This is the time when the polarization (Figure 1c) switched from right to left-handed. As the amplitude of the Pcl wave increased again as the L-mode EMIC wave, the rising tone emission was triggered.

[38] From the condition of the absolute instability, in which the wave grows at a fixed position along the magnetic field line, we have derived the amplitude threshold condition for the nonlinear wave growth to take place in the inhomogeneous magnetic field that is approximated by a parabolic function. The triggering EMIC wave modifies the velocity distribution function of the energetic protons to

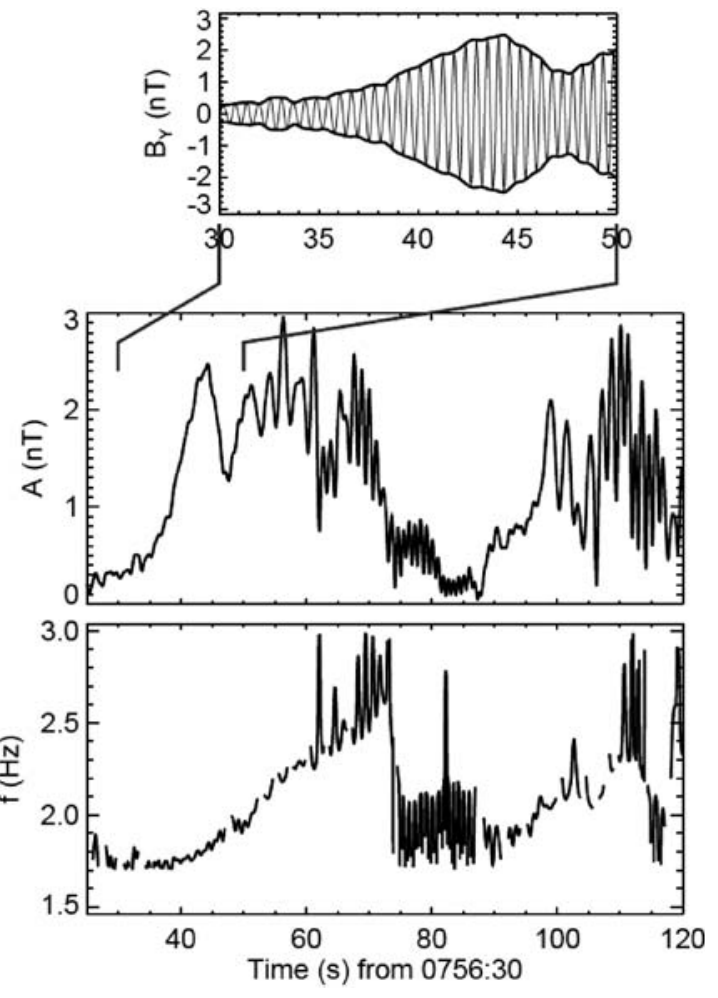

Figure 7. Amplitude and frequency analyses of the $B_{y}$ (spin plane) magnetic field waveform measured by Cluster 4 . 
(a) $[\mathrm{nT}]$

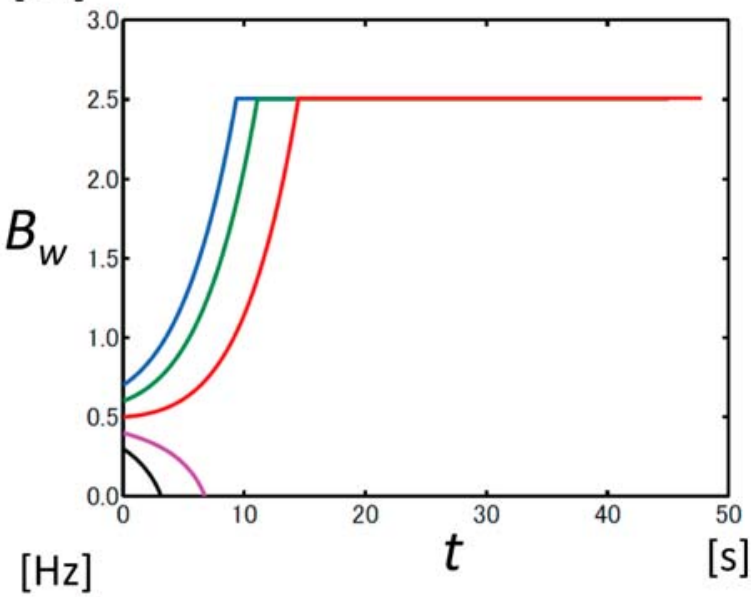

(b) $[\mathrm{Hz}]$

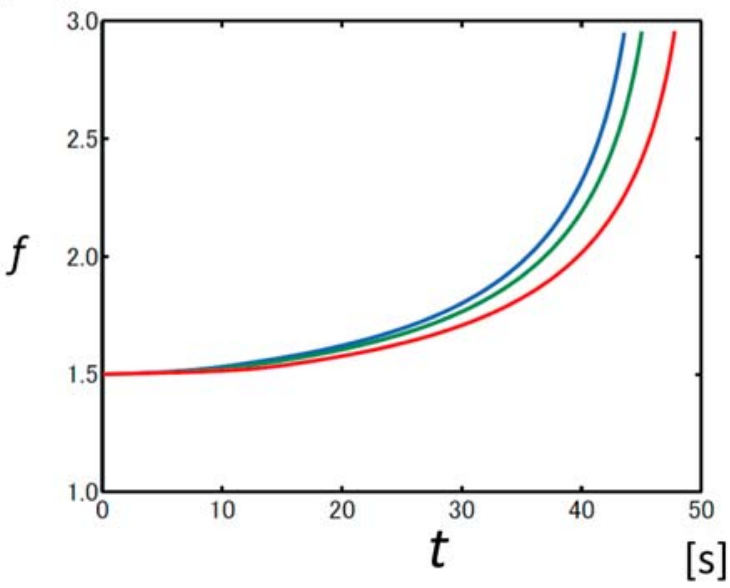

Figure 8. Solutions of the EMIC chorus equations with the wave amplitude saturation at $2.5 \mathrm{nT}$ for different initial wave amplitudes indicated by different colors: 0.3 (black), 0.4 (magenta), 0.5 (red), 0.6 (green), and 0.7 (blue) nT. (a) Wave amplitudes and (b) frequencies.

form an electromagnetic proton hole in the equatorial region. When the amplitude threshold condition is satisfied by a coherent L-mode EMIC wave component forming the proton hole, the frequency increases progressively at the magnetic equator so that the maximum nonlinear growth rate $\Gamma_{N L}$ is realized. We have shown that the coherent wave packets generated at different frequencies propagate with the group velocity at fixed frequencies.

[39] The nonlinear mechanism for the wave growth is exactly the same as for whistler-mode chorus emissions. Nonlinear growth of chorus emissions has been demonstrated to take place by using an electron hybrid simulation [Katoh and Omura, 2007; Omura et al., 2008], and a full-particle simulation [Hikishima et al., 2009a, 2009b]. The seeds of EMIC triggered emissions are generated at the magnetic equator, and the wave packets propagate with the group velocity. The nonlinear wave growth is driven by the frequency sweep rate $\partial f / \partial t$ around the magnetic equator. Beyond a critical distance $h_{c}$, given by (71), the nonlinear wave growth is driven by the spatial inhomogeneity $\partial B_{0} / \partial h$.
[40] We have derived the EMIC chorus equations that describe the variation of the wave amplitude and frequency at the magnetic equator. Using the ambient magnetic field intensity, the electron density, and the wave spectra measured by the Cluster spacecraft, we first determined the densities of $\mathrm{H}^{+}, \mathrm{He}^{+}$, and $\mathrm{O}^{+}$ions so that the wave dispersion relation agrees with the cut-off frequency of the observed wave spectra. Based on the dispersion relation, we calculated the resonance velocity of the triggering EMIC wave at $f=1.5 \mathrm{~Hz}$. Taking into account the resonance velocity, we estimated the average perpendicular velocity $V_{\perp 0}$, and the parallel thermal velocity $V_{t \|}$ from the particle measurement of energetic protons. Using these parameters, we solved the EMIC chorus equations for different initial wave amplitudes. In the solution we also assumed that the proton hole in the phase space stays in the negative range of the parallel velocity. This gives the saturation of the wave amplitude. However, the time scale of the rising tones did not agree with that of the observed rising emissions.

[41] In the detailed analysis of the wave phase and amplitude of the observed wave form, we found that the nonlinear growth of the wave amplitude saturates at $\sim 2.5 \mathrm{nT}$ as shown in Figure 7 . We reintegrated the EMIC chorus equations incorporating the observed saturation level of $2.5 \mathrm{nT}$, and found a slower variation of the wave frequency that agrees well with the observation. The exponential wave growth for $\sim 15$ seconds and the gradual frequency increase over 40 seconds found in the case presented in Figure 7 are well reproduced as the solution of the EMIC chorus equations in Figure 8.

[42] The saturation mechanism can be explained by the decreasing resonance velocity due to the increasing wave frequency of the emission. The particle velocity distribution function may not be represented properly by an assumed Maxwellian in the lower parallel velocity range. Because of the acceleration of trapped resonant particles to higher pitch angles, as observed for resonant electrons in the whistlermode chorus emission [Hikishima et al., 2009b], the proton hole may well be filled by these accelerated resonant protons at the lower resonance velocity. With the proton hole being filled, $Q$ in (54) and (63) approaches zero, quenching the nonlinear wave growth. There may also be an effect of oblique propagation, because the polarization analysis of the EMIC triggered emission as shown in Figure 1 reveals that there arises a significant compressional component $B_{w \|}$ $\sim 0.26 B_{w}$ at the end of the nonlinear growth, while the constant Pc1 wave $(\sim 1.5 \mathrm{~Hz})$ remains almost purely transverse. Such effects of the varying $Q$ value and the oblique propagation on the nonlinear wave growth are beyond the scope of the present theoretical analysis, and it is left as a future study.

[43] Acknowledgments. This work was partially supported by grantin-aid 20340135 and 17GS0208 for Creative Scientific Research "The Basic Study of Space Weather Prediction" of the Ministry of Education, Science, Sports and Culture of Japan. We acknowledge Nicole Cornilleau-Wehrlin, PI of the STAFF-SC instrument, and the Cluster Active Archive (CAA) for supplying respectively the magnetic and electric field fluctuation data. J.P. acknowledges the support of NASA Goddard Space Flight Center grant NNX07AI24G. B.G. and O.S. acknowledge support of grants GACR P205/10/2279 and GAAV A301120601. M.E. acknowledges support at Augsburg College from U.S. National Science Foundation grant ATM-0827903. 
[44] Robert Lysak thanks Andrei Demekhov and another reviewer for their assistance in evaluating this paper.

\section{References}

Anderson, B. J., R. E. Erlandson, and L. J. Zanetti (1992a), A statistical study of Pc 1-2 magnetic pulsations in the equatorial magnetosphere: 1. Equatorial occurrence distributions, J. Geophys. Res., 97, 3075-3088. Anderson, B. J., R. E. Erlandson, and L. J. Zanetti (1992b), A statistical study of Pc 1-2 magnetic pulsations in the equatorial magnetosphere: 2. Wave properties, J. Geophys. Res., 97, 3089-3101.

Arnoldy, R. L., et al. (2005), Pc 1 waves and associated unstable distributions of magnetospheric protons observed during a solar wind pressure pulse, J. Geophys. Res., 110, A07229, doi:10.1029/2005JA011041.

Cornilleau-Wehrlin, N., et al. (2003), First results obtained by the Cluster STAFF experiment, Ann. Geophys., 21, 437-456.

Engebretson, M. J., et al. (2007), Cluster observations of Pc 1-2 waves and associated ion distributions during the October and November $2003 \mathrm{mag}-$ netic storms, Planet. Space Sci., 55, 829-848, doi:10.1016/j. pss.2006.03.015.

Erlandson, R. E., L. J. Zanetti, T. A. Potemra, L. P. Block, and G. Holmgren (1990), Viking magnetic and electric field observations of Pc 1 waves at high latitudes, J. Geophys. Res., 95, 5941-5955.

Erlandson, R. E., K. Mursula, and T. Bosinger (1996), Simultaneous ground-satellite observations of structured Pc 1 pulsations, J. Geophys. Res., 101, 27,149-27,156.

Fraser, B. J., T. M. Loto'aniu, and H. J. Singer (2006), Electromagnetic ion cyclotron waves in the magnetosphere, in Magnetospheric ULF Waves. Synthesis and New Directions, Geophys. Monogr. Ser., vol. 169, edited by K. Takahashi et al., pp. 195-212, AGU, Washington, D. C.

Gendrin, R., M. Ashour-Abdalla, Y. Omura, and K. Quest (1984), Linear analysis of ion cyclotron interaction in a multicomponent plasma, J. Geophys. Res., 89, 9119-9124.

Gustafsson, G., et al. (2001), First results of electric field and density observations by Cluster EFW based on initial months of operation, Ann. Geophys., 19, 1219-1240.

Hikishima, M., S. Yagitani, Y. Omura, and I. Nagano (2009a), Full particle simulation of whistler-mode rising chorus emissions in the magnetosphere, J. Geophys. Res., 114, A01203, doi:10.1029/2008JA013625.

Hikishima, M., S. Yagitani, Y. Omura, and I. Nagano (2009b), Coherent nonlinear scattering of energetic electrons in the process of whistler mode chorus generation, J. Geophys. Res., 114, A10205, doi:10.1029/ 2009JA014371.

Kangas, J., A. Guglielmi, and O. Pokhotelov (1998), Morphology and physics of short-period magnetic pulsations, A review, Space Sci. Rev., $83,425-512$.

Katoh, Y., and Y. Omura (2007), Computer simulation of chorus wave generation in the Earth's inner magnetosphere, Geophys. Res. Lett., 34, L03102, doi:10.1029/2006GL028594.

Lorentzen, K. R., M. P. McCarthy, G. K. Parks, J. E. Foat, R. M. Millan, D. M. Smith, R. P. Lin, and J. P. Treilhou (2000), Precipitation of relativistic electrons by interaction with electromagnetic ion cyclotron waves, J. Geophys. Res., 105, 5381-5389.

Loto'aniu, T. M., B. J. Fraser, and C. L. Waters (2005), Propagation of electromagnetic ion cyclotron wave energy in the magnetosphere, J. Geophys. Res., 110, A07214, doi:10.1029/2004JA010816.

Mursula, K. (2007), Satellite observations of Pc 1 pearl waves: The changing paradigm, J. Atmos. Sol. Terr. Phys., 69, 1623-1634.

Mursula, K., L. G. Blomberg, P. A. Lindqvist, G. T. Marklund, T. Braysy, R. Rasinkangas, and P. Tanskanen (1994), Dispersive Pc1 bursts observed by Freja, Geophys. Res. Lett., 21, 1851-1854.

Mursula, K., R. Rasinkangas, and T. Bosinger (1997), Nonbouncing Pc 1 wave bursts, J. Geophys. Res., 102, 17,611-17,624.

Mursula, K., T. Braysy, K. Niskala, and C. T. Russell (2001), Pc1 pearls revisited: Structured electromagnetic ion cyclotron waves on Polar satellite and on the ground, J. Geophys. Res., 106, 29,543-29,533.

Omura, Y., and H. Matsumoto (1982), Computer simulations of basic processes of coherent whistler wave-particle interactions in the magnetosphere, J. Geophys. Res., 87, 4435-4444.

Omura, Y., and D. Summers (2006), Dynamics of high-energy electrons interacting with whistler mode chorus emissions in the magnetosphere, J. Geophys. Res., 111, A09222, doi:10.1029/2006JA011600.
Omura, Y., Y. Katoh, and D. Summers (2008), Theory and simulation of the generation of whistler-mode chorus, J. Geophys. Res., 113, A04223, doi:10.1029/2007JA012622.

Omura, Y., M. Hikishima, Y. Katoh, D. Summers, and S. Yagitani (2009), Nonlinear mechanisms of lower-band and upper-band VLF chorus emissions in the magnetosphere, J. Geophys. Res., 114, A07217, doi:10.1029/ 2009JA014206.

Pickett, J. S., et al. (2010), Cluster observations of EMIC triggered emissions in association with Pc1 waves near Earth's plasmapause, Geophys. Res. Lett., 37, L09104, doi:10.1029/2010GL042648.

Rème, H., et al. (2001), First multispacecraft ion measurements in and near the Earth's magnetosphere with the identical Cluster ion spectrometry (CIS) experiment, Ann. Geophys., 19, 1303-1354, doi:10.5194/angeo19-1303-2001.

Roux, A., S. Perraut, J. Rauch, C. de Villedary, G. Kremser, A. Korth, and D. Young (1982), Wave-particle interactions near $\Omega_{\mathrm{He}}$ observed on board GEOS 1 and 2: 2. Generation of ion cyclotron waves and heating of $\mathrm{He}+$ ions, J. Geophys. Res., 87, 8174-8190.

Santolik, O., and D. A. Gurnett (2002), Propagation of auroral hiss at high altitudes, Geophys. Res. Lett., 29(10), 1481, doi:10.1029/2001GL013666. Santolik, O., F. Lefeuvre, M. Parrot, and J. L. Rauch (2001), Complete wave-vector directions of electromagnetic emissions: Application to INTERBALL-2 measurements in the nightside auroral zone, J. Geophys. Res., 106, 13,191-13,201.

Santolik, O., J. S. Pickett, D. A. Gurnett, and L. R. O. Storey (2002), Magnetic component of narrowband ion cyclotron waves in the auroral zone, J. Geophys. Res., 107(A12), 1444, doi:10.1029/2001JA000146.

Santolik, O., M. Parrot, and F. Lefeuvre (2003a), Singular value decomposition methods for wave propagation analysis, Radio Sci., 38(1), 1010, doi:10.1029/2000RS002523.

Santolik, O., D. A. Gurnett, J. S. Pickett, M. Parrot, and N. CornilleauWehrlin (2003b), Spatio-temporal structure of storm-time chorus, J. Geophys. Res., 108(A7), 1278, doi:10.1029/2002JA009791.

Summers, D., and R. M. Thorne (2003), Relativistic electron pitch-angle scattering by electromagnetic ion cyclotron waves during geomagnetic storms, J. Geophys. Res., 108(A4), 1143, doi:10.1029/2002JA009489.

Summers, D., B. Ni, and N. P. Meredith (2007), Timescales for radiation belt electron acceleration and loss due to resonant wave-particle interactions: 2. Evaluation for VLF chorus, ELF hiss, and electromagnetic ion cyclotron waves, 112, A04207, doi:10.1029/2006JA011993.

Tepley, L. R. (1961), Observations of hydromagnetic emissions, J. Geophys. Res., 66, 1651-1658.

Trakhtengerts, V. Y., and A. G. Demekhov (2007), Generation of Pc 1 pulsations in the regime of backward wave oscillator, J. Atmos. Sol. Terr. Phys, 69, 1651-1656.

Trakhtengerts, V. Y., and M. J. Rycroft (2008), Whistler and Alfven Mode Cyclotron Masers in Space, Cambridge Univ. Press, Cambridge, Mass. Troitskaya, V. A. (1961), Pulsations of the earth's electromagnetic field with periods of 1 to 1.5 seconds and their connection with phenomena in the high atmosphere, J. Geophys. Res., 66, 5-18.

I. Dandouras, Centre d'Etude Spatiale des Rayonnements, Université de Toulouse, 9 Ave. du Colonel Roche, BP 44346, F-31028 Toulouse CEDEX 4, France. (iannis.dandouras@cesr.fr)

P. M. E. Décréau, Laboratoire de Physique et Chimie de l'Environnement et de l'Espace, UMR 6115, Université d'Orléans, CNRS, 3A Ave. de la Recherche Scientifique, F-45071 Orleans CEDEX 2, France. (pierrette. decreau@cnrs-orleans.fr)

M. Engebretson, Department of Physics, Augsburg College, 2211 Riverside Ave., Minneapolis, MN 55454, USA. (engebret@augsburg.edu)

B. Grison and O. Santolik, Institute of Atmospheric Physics, Academy of Sciences of the Czech Republic, Bocní II 1401, 14131 Prague 4, Czech Republic. (grison@ufa.cas.cz; os@ufa.cas.cz)

A. Masson, Science Operations Department, European Space Agency, Keplerlaan 1, NL-2201 AZ, Noordwijk, Netherlands. (amasson@rssd.esa. int)

Y. Omura, Research Institute for Sustainable Humanosphere, Kyoto University, Uji, Kyoto 611-0011, Japan. (omura@rish.kyoto-u.ac.jp)

J. Pickett, Department of Physics and Astronomy, University of Iowa, Iowa City, IA 52242, USA. (pickett@uiowa.edu) 